\title{
Tobacco smoking and the risk of gallbladder disease
}

\author{
Dagfinn Aune $^{1,2} \cdot$ Lars J. Vatten ${ }^{1} \cdot$ Paolo Boffetta $^{3}$
}

Received: 24 August 2015/Accepted: 13 January 2016/Published online: 22 February 2016

(c) The Author(s) 2016. This article is published with open access at Springerlink.com

\begin{abstract}
Tobacco smoking has been inconsistently associated with gallbladder disease risk. To clarify the association we conducted a systematic review and metaanalysis of cohort studies published on the subject. We searched the PubMed and Embase databases for studies of smoking and gallbladder disease up to January 9th 2015. Prospective studies were included if they reported relative risk estimates and $95 \%$ confidence intervals of gallbladder disease associated with current, former or ever smoking and by number of cigarettes per day. Summary relative risks were estimated by use of a random effects model. We identified ten prospective studies including 59,530 gallbladder disease cases among 4,213,482 participants that could be included in the meta-analysis. The summary RR was $1.19\left(95 \%\right.$ CI $\left.1.12-1.28, \mathrm{I}^{2}=46.9 \%, \mathrm{n}=6\right)$ for current smokers, 1.10 (95\% CI $1.07-1.13, \mathrm{I}^{2}=0 \%$, $\mathrm{n}=6$ ) for former smokers and 1.15 (95\% CI 1.13-1.18, $\left.\mathrm{I}^{2}=0 \%, \mathrm{n}=7\right)$ for ever smokers. In the dose-response analysis the summary relative risk was 1.11 (95\% CI $1.08-1.14, \mathrm{I}^{2}=33 \%, \mathrm{n}=3$ ) per 10 cigarettes per day and
\end{abstract}

Electronic supplementary material The online version of this article (doi:10.1007/s10654-016-0124-z) contains supplementary material, which is available to authorized users.

Dagfinn Aune

d.aune@imperial.ac.uk

1 Department of Public Health and General Practice, Faculty of Medicine, Norwegian University of Science and Technology, Trondheim, Norway

2 Department of Epidemiology and Biostatistics, School of Public Health, Imperial College London, St. Mary's Campus, Norfolk Place, Paddington, London W2 1PG, UK

3 Institute of Translational Epidemiology and Tisch Cancer Institute, Icahn School of Medicine at Mount Sinai, New York, NY, USA although there was indication of nonlinearity there was a dose-dependent positive association with increasing number of cigarettes smoked per day. The current meta-analysis provides evidence of an increased risk of gallbladder disease associated with tobacco smoking.

Keywords Tobacco smoking · Gallstones ·

Cholelithiasis · Cholecystectomy · Gallbladder disease .

Cohort studies · Meta-analysis

\section{Introduction}

Gallbladder disease is a major cause of morbidity in the US and in the Europe. The prevalence of asymptomatic gallbladder disease ranges between 10-30\% within these populations [1], while symptomatic gallbladder disease is less frequent and affects approximately $2 \%$ of the population [2]. Of digestive diseases that require hospitalization gallbladder disease is the most frequent and costly; the economic costs of hospital treatment of gallbladder disease is over 5 billion US dollar per year [3, 4].

Tobacco smoking is believed to affect the hepatobiliary system and has been associated with increased risk of liver [5] and gallbladder cancer [6]. Studies of smoking in relation to gallbladder disease and gallstones risk have, however, been mixed. Several case-control and crosssectional studies did not find an association [7-11] or even inverse associations [12], while a few did report increased risk [13, 14], although not always significantly so. However, it is possible that such studies may have been affected by recall bias, selection bias or temporal biases, thus it's difficult to draw conclusions based on these study designs. Prospective studies, which are less prone to such biases, have also been inconsistent with some studies showing no 
significant association $[15,16]$, while some larger studies did report a moderate increase in risk associated with tobacco smoking [17-24], and some [17, 20, 21] of these suggested a dose-response relationship with increasing number of cigarettes smoked per day. We conducted a systematic review and meta-analysis of cohort studies on the association between smoking and gallbladder disease with the aim of clarifying whether there is an association, the strength of any potential association and if there is a dose-response relationship between smoking and gallbladder disease.

\section{Methods}

\section{Search strategy and inclusion criteria}

We developed a systematic review protocol for the project. Pubmed and Embase databases were searched up to January 9th 2015 for eligible studies. As part of a larger project on risk factors for gallbladder disease we used wide search terms PubMed search: (body mass index OR BMI OR overweight OR obesity OR anthropometry OR fatness OR body fatness OR abdominal fatness OR abdominal

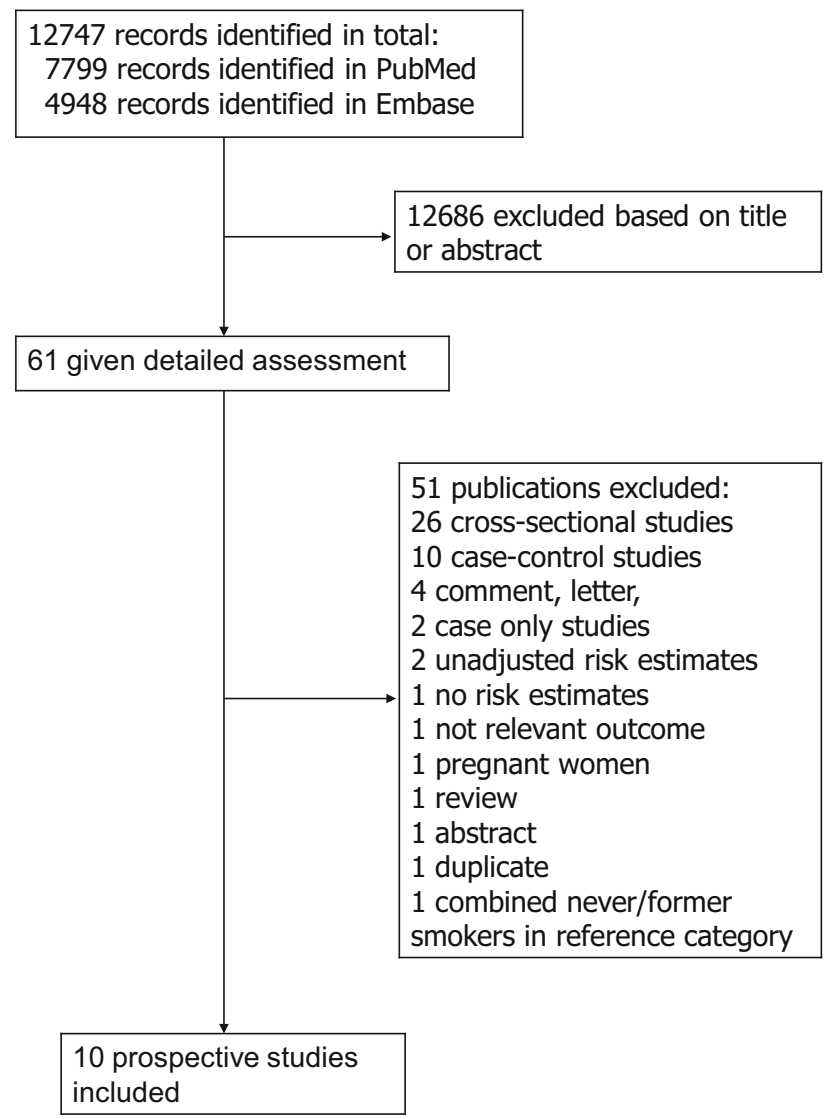

Fig. 1 Flow-chart of study selection obesity OR waist circumference OR waist-to-hip ratio OR physical activity OR exercise OR sports OR walking OR biking OR running OR fitness OR exercise test OR inactivity OR diabetes OR smoking OR tobacco OR risk factor OR risk factors) AND (gallstones OR gallbladder disease OR cholelithiasis OR cholecystectomy OR cholecystitis). We followed standard criteria for conducting and reporting meta-analyses [25]. In addition, we searched the reference lists of the identified publications for further studies. One reviewer (DA) conducted the initial screening of all the references and two reviewers (DA, LJV) conducted the screening of the potentially eligible studies. Any disagreements were resolved by discussion between the authors.

\section{Study selection}

We included published prospective studies that investigated the association between smoking and the risk of gallbladder disease, gallstones, or cholecystectomies. Adjusted estimates of the relative risk had to be available with the $95 \%$ CIs in the publication. For the dose-response analysis a quantitative measure of the smoking level had to be provided. We identified ten relevant prospective studies that could be included in the analysis [15-24]. A list of the excluded studies and the reason for exclusion is provided in Supplementary Table 1.

\section{Data extraction}

The following data were extracted from each study: The first author's last name, publication year, country where the study was conducted, study period, sample size, sex, number of cases, smoking type, cigarettes per day, relative risks and $95 \%$ confidence intervals for the highest versus the lowest level of smoking and variables adjusted for in the analysis. One reviewer extracted the data (DA) and they were checked for accuracy by a second reviewer (LJV). Any disagreements were resolved by discussion.

\section{Statistical methods}

We calculated summary relative risks for the highest versus the lowest level of smoking using the random-effects model by DerSimonian and Laird [26] which takes into account both within and between study variation (heterogeneity). The average of the natural logarithm of the relative risks was estimated and the relative risk from each study was weighted by the inverse of its variance.

To investigate whether the number of cigarettes smoked per day was associated with gallbladder disease we used the method described by Greenland and Longnecker [27] to conduct dose-response analysis by 


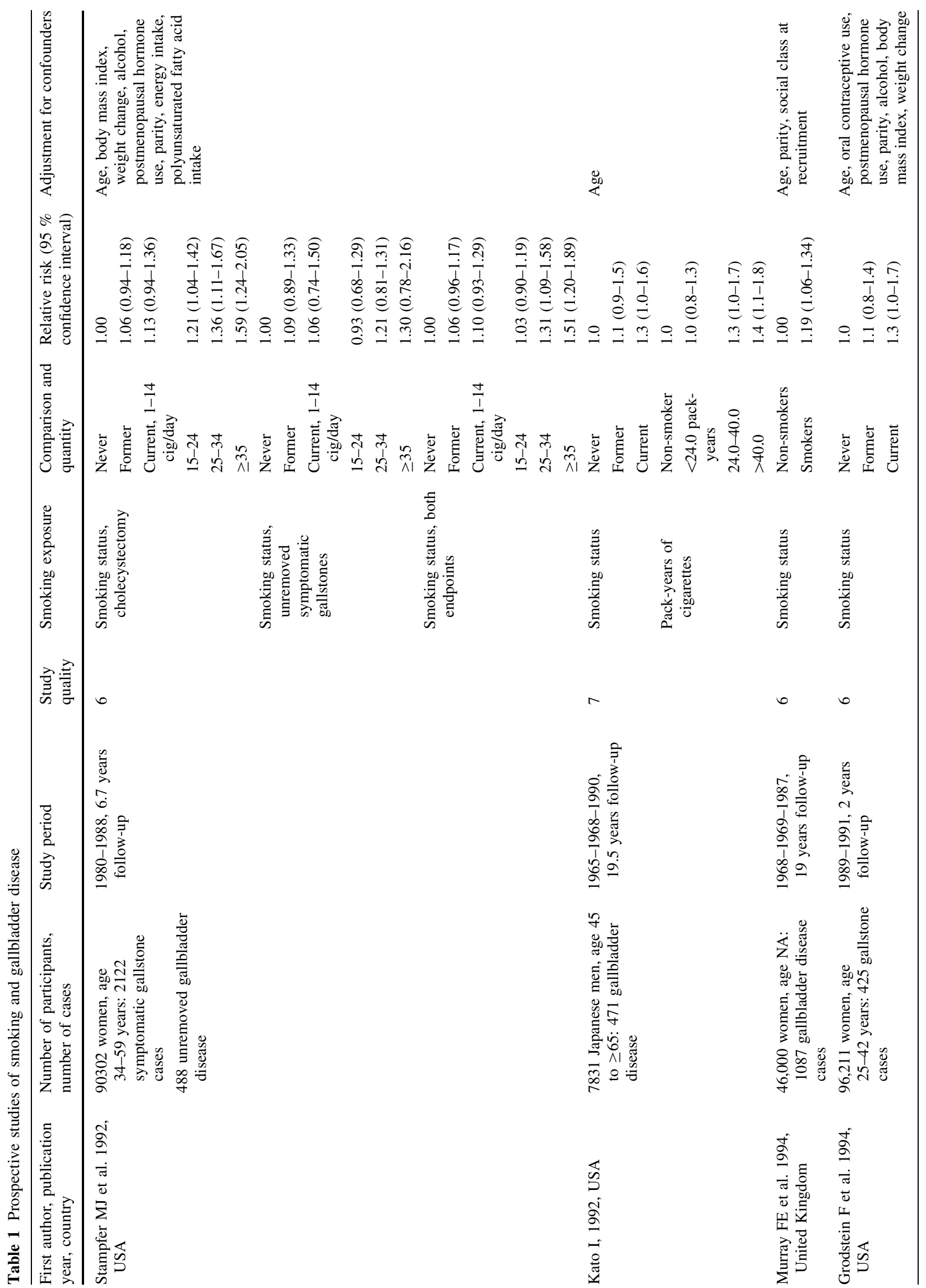




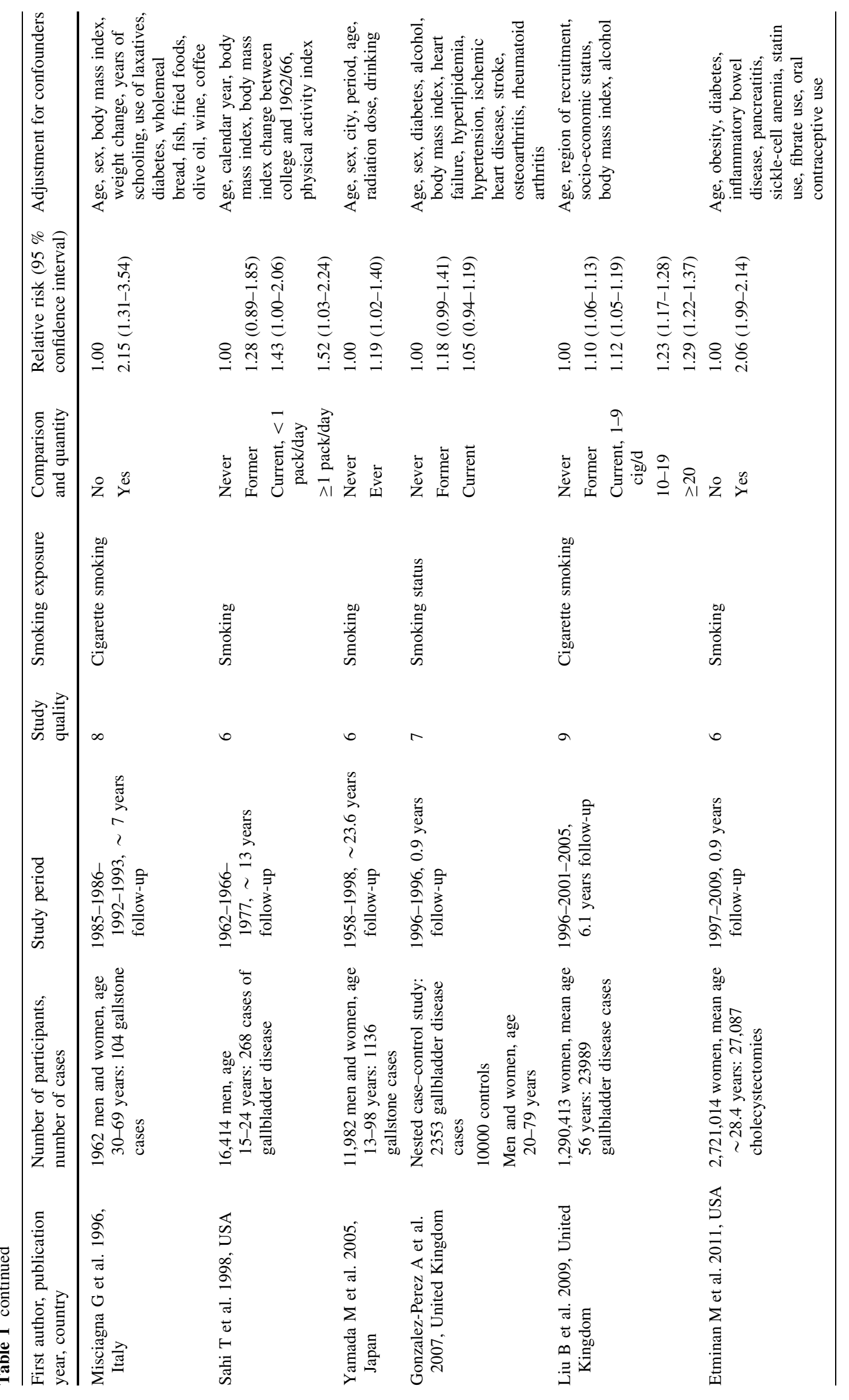


Fig. 2 Current smokers (a) and former smokers (b) versus never smokers and gallbladder disease

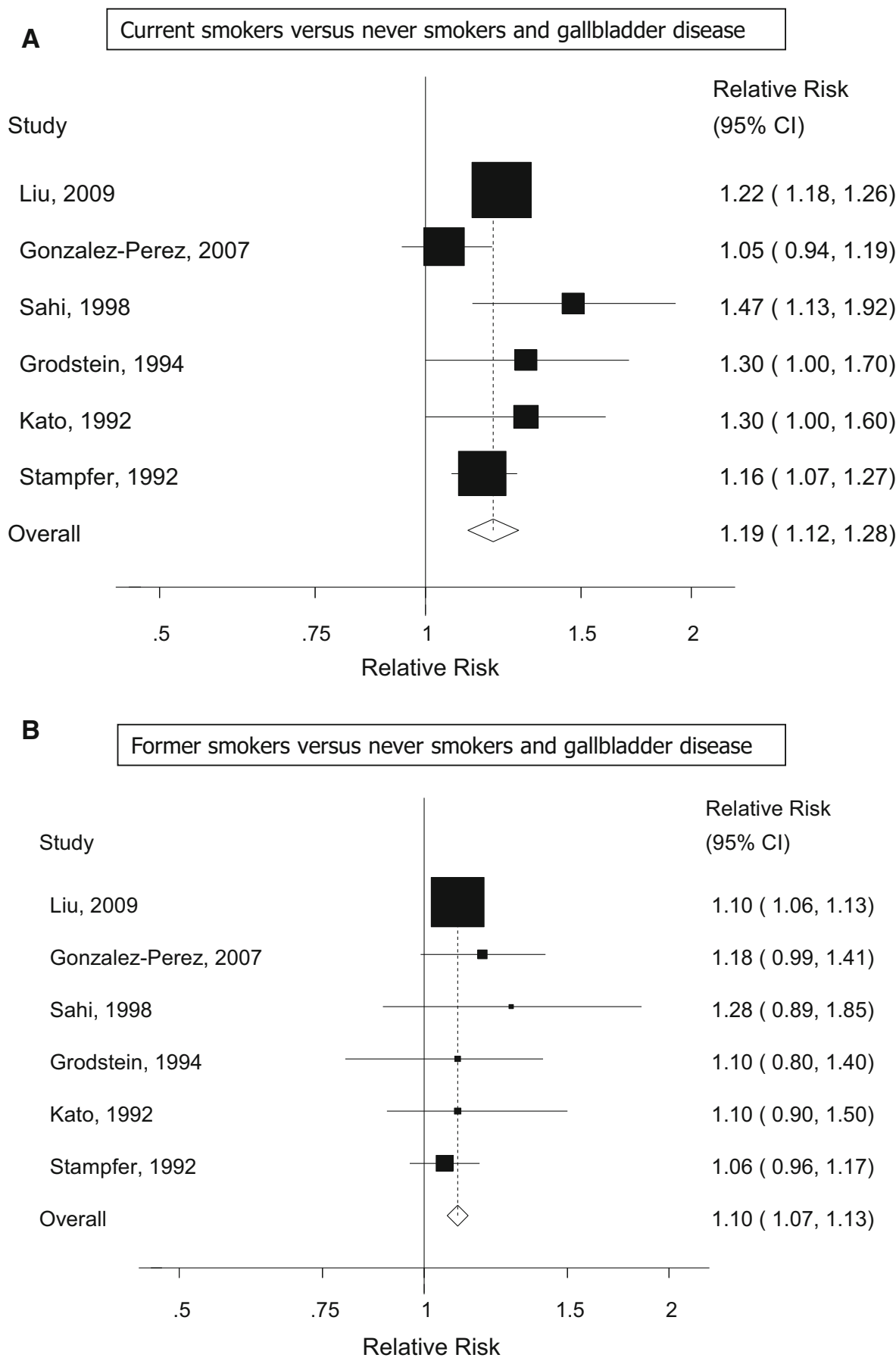

computing study-specific slopes (linear trends) and $95 \%$ confidence intervals from the natural $\log$ of the relative risks and confidence intervals across categories of cigarettes per day. The method requires that the distribution of cases and person-years or non-cases and the relative risks with the variance estimates for at least three quantitative exposure categories are known. For studies that did not provide this information, we estimated the distribution of cases and person-years or non- cases based on a method previously described [28]. Studies that did not quantify the number of cigarettes smoked per day were excluded from the dose-response analysis. We assessed a potential nonlinear dose-response relationship between smoking and gallbladder disease using fractional polynomial models. We determined the best fitting second order fractional polynomial regression model, defined as the one with the lowest deviance. A likelihood ratio test was used to assess the 
Fig. 3 Ever smokers versus never smokers and gallbladder disease

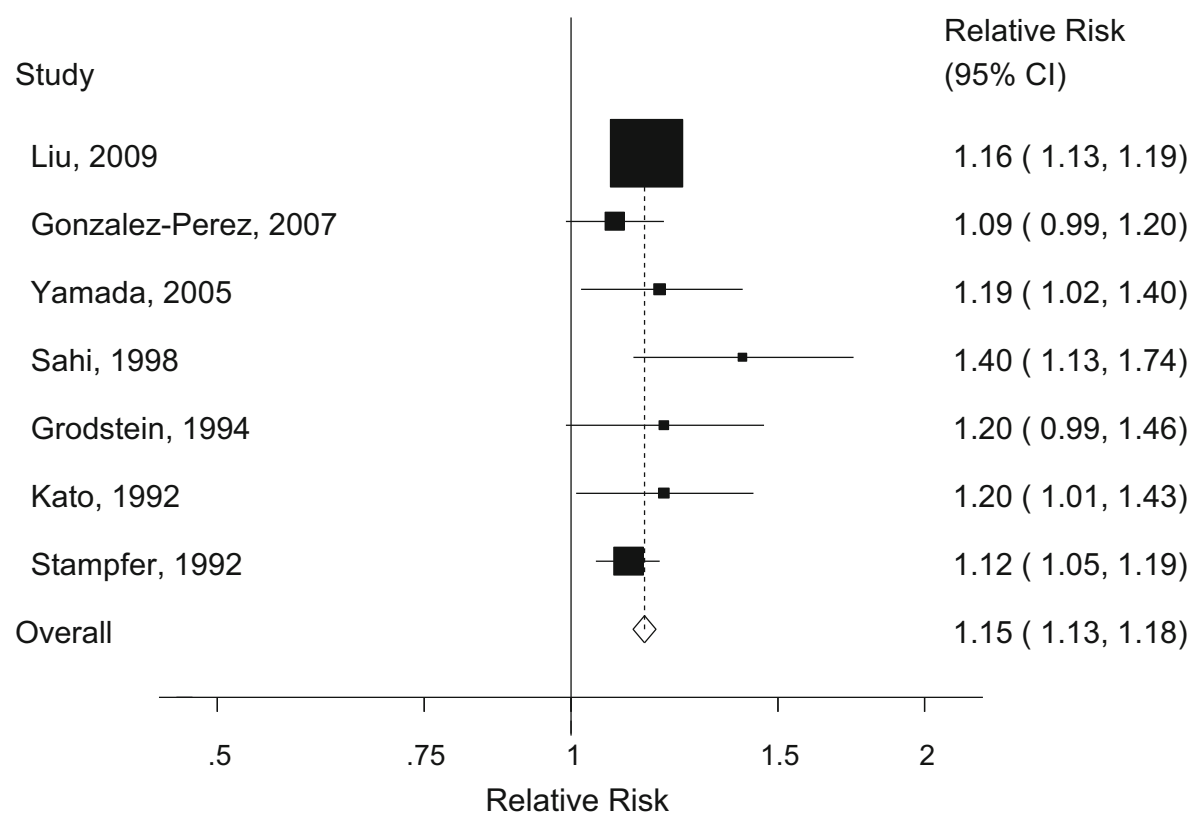

difference between the nonlinear and linear models to test for nonlinearity [29].

Heterogeneity between studies was evaluated using Q and $\mathrm{I}^{2}$ statistics [30]. All statistical tests were two-sided and $p<0.05$ considered statistically significant. $\mathrm{I}^{2}$-values of 25, 50 and $75 \%$ indicates low, moderate and high heterogeneity, respectively [31]. We conducted main analyses (all studies combined) and stratified by study characteristics such as sample size, number of cases, geographic location, study quality score and by adjustment for confounding factors. Study quality was assessed using the Newcastle-Ottawa scale which ranks the studies on a scale from 0 to 9 based on the selection of the study population, comparability between cases and non-cases and the assessment of the outcome [32].

Publication bias was assessed using Egger's test [33] and Begg-Mazumdar's test [34] and with funnel plots, and $p<0.10$ was considered to indicate possible publication bias as the tests have low power when the number of studies is low. The statistical analyses were conducted using the software package Stata, version 9.0 software (StataCorp, Texas, US).

\section{Results}

Out of a total of 12,747 records identified by the searches, we identified 10 prospective studies [15-24] involving a total of 61,071 cases among 4,344,553 participants that could be included in the analyses of smoking and gallbladder disease (Fig. 1, Table 1). Five of the studies were from North-America, four were from Europe and one was from Asia (Table 1).

\section{Smoking status}

Six cohort studies [16-21] were included in the analysis of current smoking and gallbladder disease and included 30,533 cases among 1,513,524 participants. The summary RR was 1.19 (95 \% CI 1.12-1.28, $\mathrm{I}^{2}=46.9 \%$, pheterogeneity $=0.09$ ) (Fig. 2a). Six cohort studies [16-21] were included in the analysis of former smoking and gallbladder disease (30,533 cases and 1,513,524 participants) and the summary RR was 1.10 (95\% CI 1.07-1.13, $\mathrm{I}^{2}=0 \%$, $\mathrm{p}_{\text {heterogeneity }}=0.88$ ) (Fig. 2b). Seven cohort studies [16-21, 24] were included in the analysis of ever smoking and gallbladder disease $(31,669$ cases and 1,525,506 participants) and the summary RR was 1.15 (95\% CI 1.13-1.18, $\mathrm{I}^{2}=0 \%$, pheterogeneity $=0.43$ ) (Fig. 3). Three cohort studies [15, 22, 23] with 28,278 cases and 2768976 participants, where the smoking status or the reference category was not clearly defined, were analyzed separately and gave a summary RR of 1.70 (95\% CI 1.09-2.67, $\mathrm{I}^{2}=97 \%$, pheterogeneity $<0.0001$ ) (Supplementary Figure 1). There was no evidence of publication bias in the analysis of current smokers, $p=0.98$ and $p=0.99$ with Egger's test and with Begg's test respectively, former smokers, $p=0.46$ and $p=0.71$, or ever smokers, $p=0.58$ and $p=0.37$, respectively, although there was possibly slight asymmetry in the funnel plots (Supplementary Figures 2-4). However, this was driven by one or two outlying studies which did not affect the overall summary estimates. 
Fig. 4 Cigarette smoking and gallbladder disease, linear and nonlinear dose-response analyses

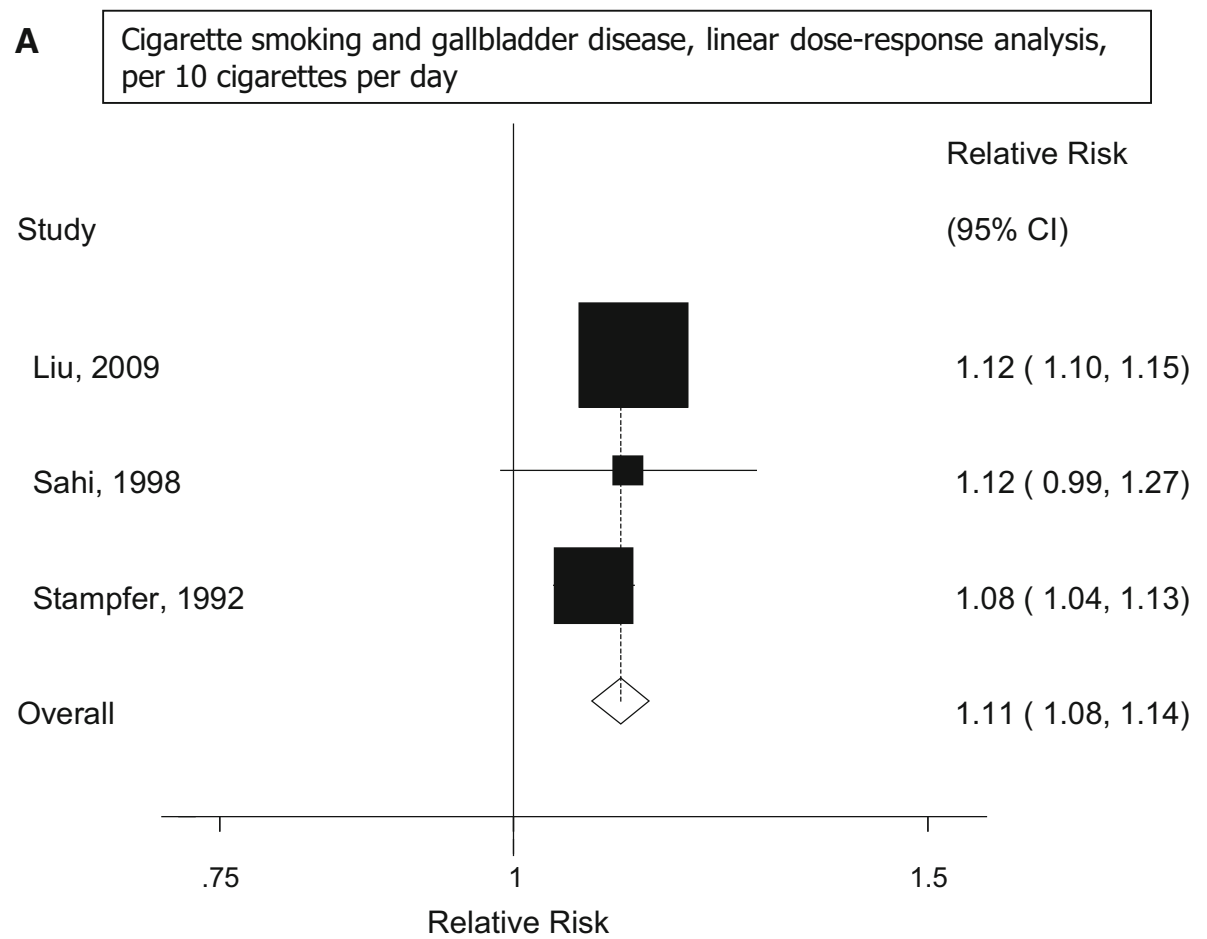

B

Cigarette smoking and gallbladder disease, nonlinear dose-response analysis

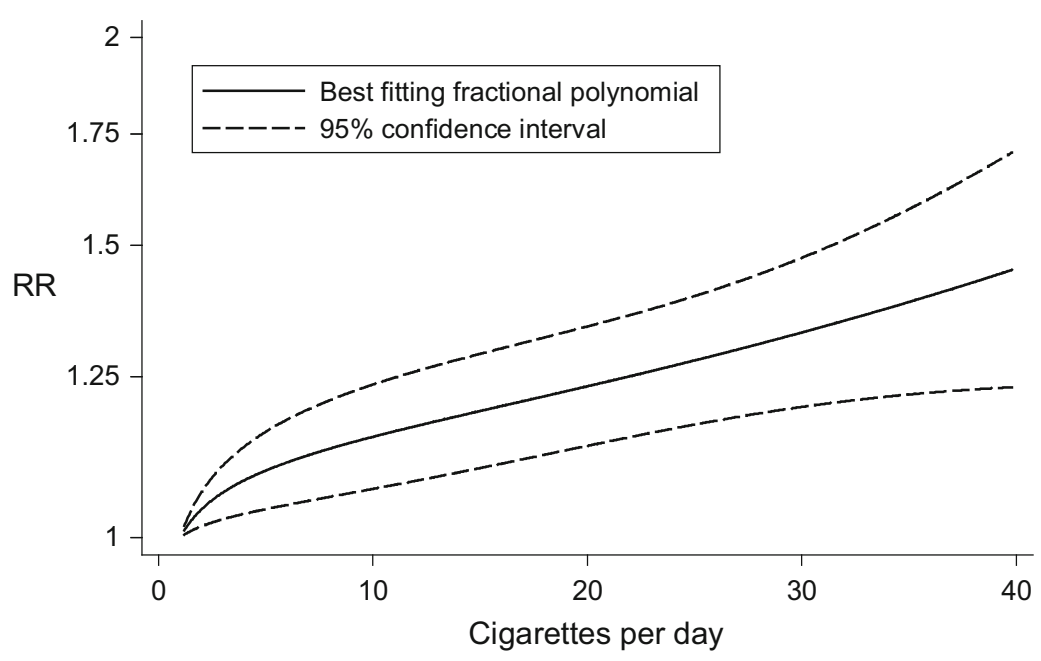

\section{Dose-response analyses}

Three cohort studies $[17,20,21]$ were included in the dose-response analysis of cigarettes per day and gallbladder disease risk. The summary relative risk was 1.11 (95\% CI $1.08-1.14, \mathrm{I}^{2}=33 \%$, pheterogeneity $=0.23$ ) per 10 cigarettes per day (Fig. 4a). There was some suggestion of a nonlinear association, pnonlinearity $<0.0001$, with a slightly steeper increase in the risk from low levels, but the association appeared to be linear from about 5 cigarettes per day (Fig. 4b).

\section{Subgroup and sensitivity analyses and cumulative meta-analyses}

In subgroup analyses there were positive associations in most strata, defined by study design, gender, outcometype, geographic location, number of cases, study quality and adjustment for confounding factors (Table 2). With metaregression analyses there was little evidence that the results differed between these subgroups (Table 2). When we conducted sensitivity analyses removing one study at a time, the size of the summary estimates were very similar 


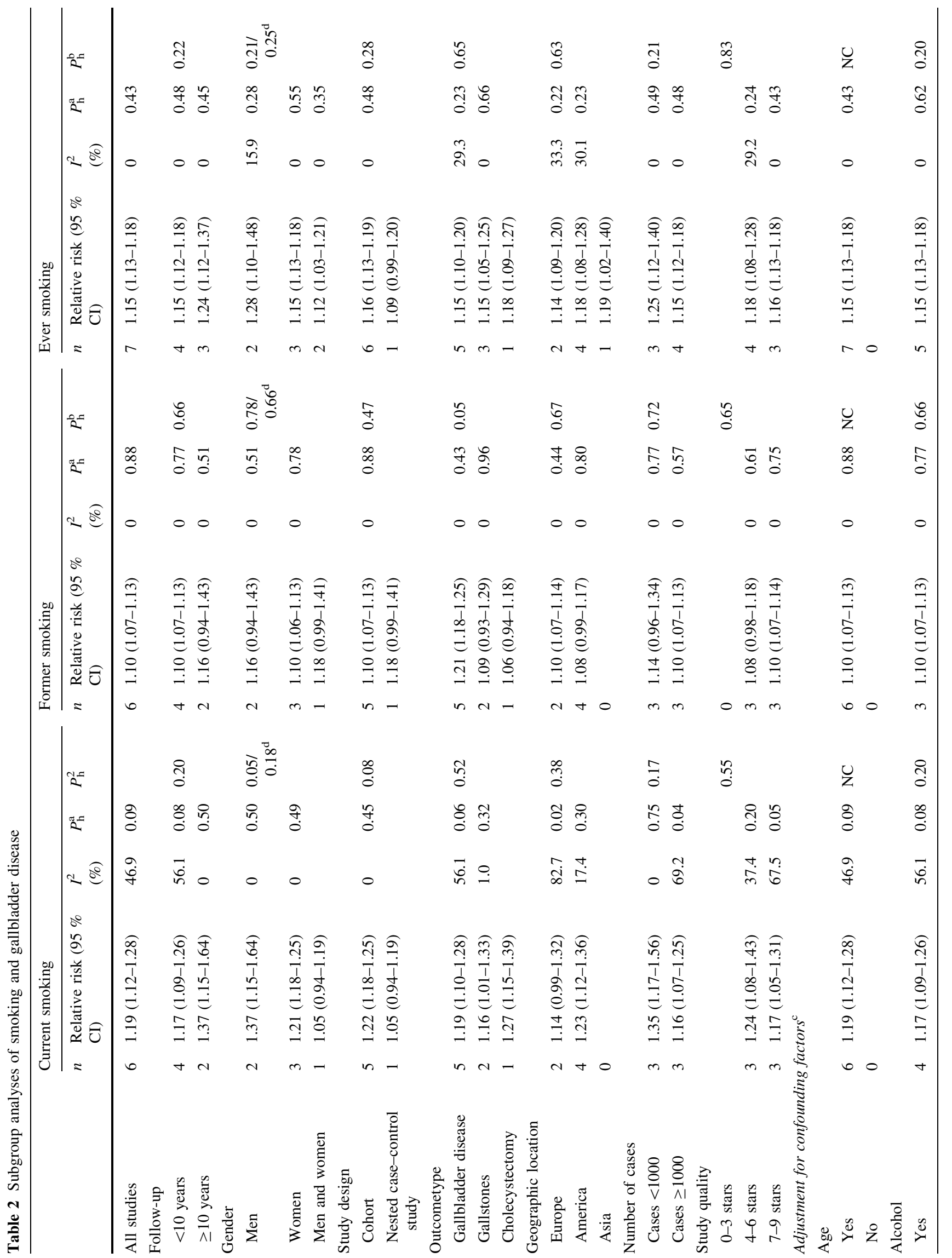




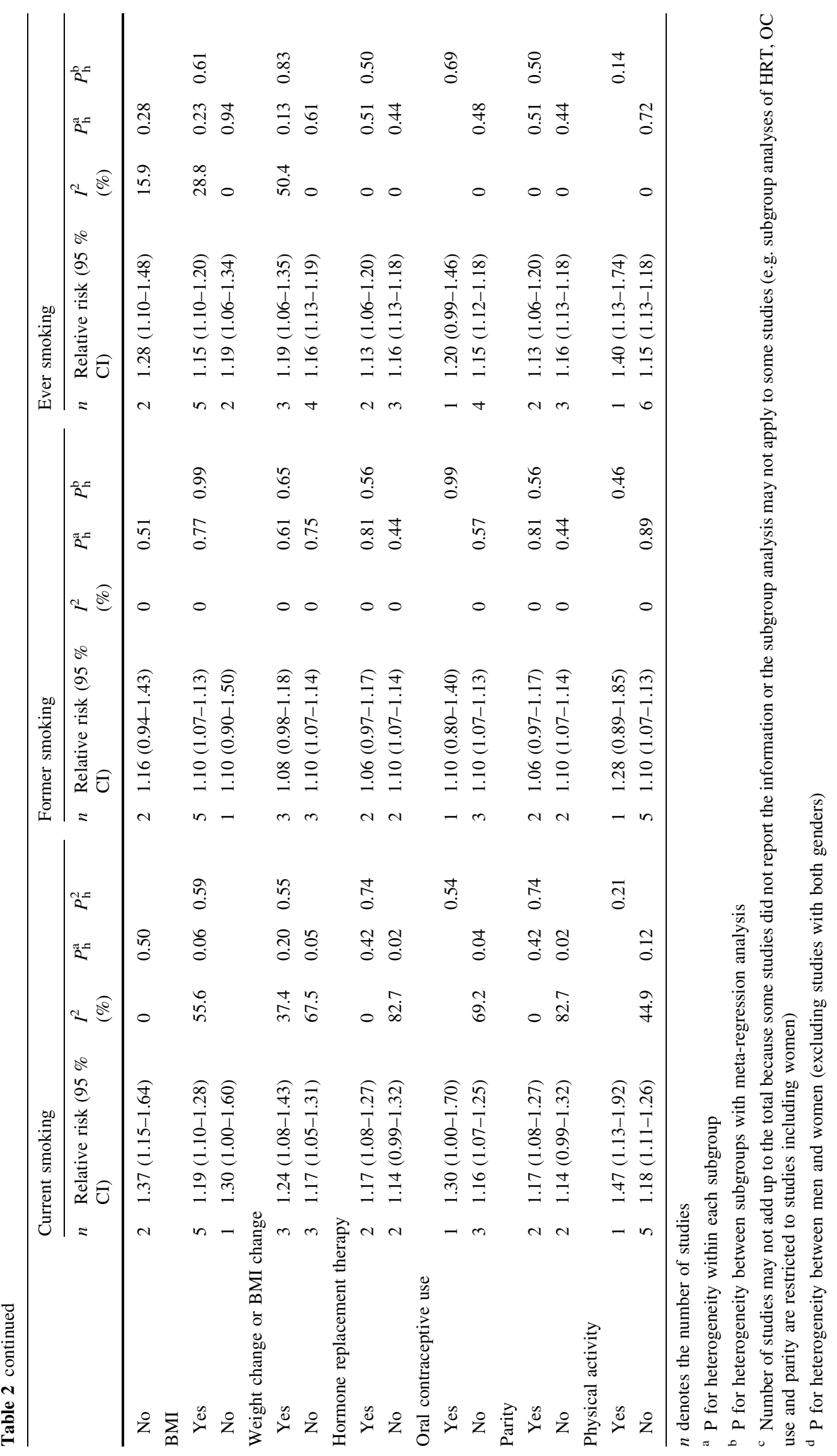


(Supplementary Figures 5-7). In cumulative meta-analyses, there was little change in the size of the summary RRs over time (Supplementary Figures 8-10), although for former smoking the association became significant in 2007, while for current and ever smoking the associations were significant from the first studies published in 1992.

\section{Discussion}

To our knowledge this is the first meta-analysis of observational studies of smoking and risk of gallbladder disease and our results confirm a $19 \%$ increased relative risk among current smokers, with a dose-response relationship of increasing risk with increasing number of cigarettes smoked per day. In addition, a 10 and $15 \%$ increase in the relative risk was observed for former and ever smokers as well.

Little is known about the biological mechanisms that could explain the adverse effect of smoking on risk of gallbladder disease. Smoking has been shown to increase the risk of type 2 diabetes [35] which is a risk factor for gallbladder disease [36]. Cigarette smoking has also been associated with increased risk of gallbladder cancer [6], although the exact mechanism is not known. In addition, smoking may increase gallbladder disease risk by reducing plasma high density lipoprotein cholesterol concentrations [37] as higher levels of HDL cholesterol are associated with lower gallbladder disease risk [38]. Tobacco smoke contains several dozens of toxic chemicals that may have detrimental effects on the gallbladder by as yet unidentified mechanisms. Further studies are needed to clarify the mechanism(s) that may explain the increased risk of gallbladder disease among smokers.

The present systematic review and meta-analysis has some limitations that need to be discussed. The number of studies included was moderate and some studies could not be included in the dose-response analysis because only smoking status and not results for number of cigarettes per day were reported. Further studies should aim to clarify the dose-response relationship between number of cigarettes per day, duration of smoking, and time since quitting smoking in relation to gallbladder disease risk and report sufficient details to be included in future updated doseresponse analyses. Many of the included studies adjusted for important confounding factors and the results persisted in subgroup analyses by whether the studies adjusted for body mass index, weight change, alcohol, hormone replacement therapy use and parity, although there were few studies in some of these subgroup analyses. Publication bias is a possibility, but we did not find evidence of such bias with the statistical tests used or by inspection of the funnel plots, although the number of studies was moderate. Strengths of the present meta-analysis include the detailed dose-response, subgroup and sensitivity analyses, and the large sample size providing a robust estimate of the association between smoking and risk of gallbladder disease.

In conclusion, the results from this systematic review and meta-analysis provide further evidence that smoking increases the risk of developing gallbladder disease. Considering the relatively few modifiable risk factors that have been established for gallbladder disease as well as the many other adverse effects of smoking, further efforts to reduce the prevalence of smoking are needed. Any further studies should report more detailed results by intensity and duration of smoking and clarify the impact of smoking cessation on gallbladder disease risk.

Acknowledgments This work was funded by "Liaison Committee between the Central Norway Regional Health Authority (RHA) and the Norwegian University of Science and Technology (NTNU)". The study sponsor had no role in the study design, collection of data, analysis, and interpretation of data. D. Aune takes primary responsibility for the integrity of the data and the accuracy of the data analysis. The authors thank Darren C. Greenwood (PhD, Biostatistics Unit, Centre for Epidemiology and Biostatistics, University of Leeds) for the Stata code for the nonlinear dose-response analysis.

\section{Compliance with ethical standards}

Conflict of interest All authors declare that there are no conflicts of interest related to this manuscript.

Open Access This article is distributed under the terms of the Creative Commons Attribution 4.0 International License (http://crea tivecommons.org/licenses/by/4.0/), which permits unrestricted use, distribution, and reproduction in any medium, provided you give appropriate credit to the original author(s) and the source, provide a link to the Creative Commons license, and indicate if changes were made.

\section{References}

1. Kratzer W, Mason RA, Kachele V. Prevalence of gallstones in sonographic surveys worldwide. J Clin Ultrasound. 1999;27:1-7.

2. Friedman GD, Raviola CA, Fireman B. Prognosis of gallstones with mild or no symptoms: 25 years of follow-up in a health maintenance organization. J Clin Epidemiol. 1989;42:127-36.

3. Shaffer EA. Gallstone disease: epidemiology of gallbladder stone disease. Best Pract Res Clin Gastroenterol. 2006;20:981-96.

4. Sandler RS, Everhart JE, Donowitz M, et al. The burden of selected digestive diseases in the United States. Gastroenterology. 2002;122:1500-11.

5. Gandini S, Botteri E, Iodice $S$, et al. Tobacco smoking and cancer: a meta-analysis. Int J Cancer. 2008;122:155-64.

6. Wenbin D, Zhuo C, Zhibing M, et al. The effect of smoking on the risk of gallbladder cancer: a meta-analysis of observational studies. Eur J Gastroenterol Hepatol. 2013;25:373-9.

7. Jorgensen T. Gall stones in a Danish population. Relation to weight, physical activity, smoking, coffee consumption, and diabetes mellitus. Gut. 1989;30:528-34. 
8. Kono S, Shinchi K, Ikeda N, Yanai F, Imanishi K. Prevalence of gallstone disease in relation to smoking, alcohol use, obesity, and glucose tolerance: a study of self-defense officials in Japan. Am J Epidemiol. 1992;136:787-94.

9. Okamoto M, Yamagata Z, Takeda Y, Yoda Y, Kobayashi K, Fujino MA. The relationship between gallbladder disease and smoking and drinking habits in middle-aged Japanese. J Gastroenterol. 2002;37:455-62.

10. Kratzer W, Kachele V, Mason RA, et al. Gallstone prevalence in relation to smoking, alcohol, coffee consumption, and nutrition. The Ulm Gallstone study. Scand J Gastroenterol. 1997;32:953-8.

11. Kono S, Eguchi H, Honjo S, et al. Cigarette smoking, alcohol use, and gallstone risk in Japanese men. Digestion. 2002;65:177-83.

12. Pastides H, Tzonou A, Trichopoulos D, et al. A case-control study of the relationship between smoking, diet, and gallbladder disease. Arch Intern Med. 1990;150:1409-12.

13. McMichael AJ, Baghurst PA, Scragg RK. A case-control study of smoking and gallbladder disease: importance of examining time relations. Epidemiology. 1992;3:519-22.

14. Kato I, Kato K, Akai S, Tominaga S. A case-control study of gallstones: a major risk factor for biliary tract cancer. Jpn J Cancer Res. 1990;81:578-83.

15. Murray FE, Logan RF, Hannaford PC, Kay CR. Cigarette smoking and parity as risk factors for the development of symptomatic gall bladder disease in women: results of the royal college of general practitioners' oral contraception study. Gut. 1994;35:107-11.

16. Gonzalez-Perez A, Garcia Rodriguez LA. Gallbladder disease in the general population: association with cardiovascular morbidity and therapy. Pharmacoepidemiol Drug Saf. 2007;16:524-31.

17. Stampfer MJ, Maclure KM, Colditz GA, Manson JE, Willett WC. Risk of symptomatic gallstones in women with severe obesity. Am J Clin Nutr. 1992;55:652-8.

18. Kato I, Nomura A, Stemmermann GN, Chyou PH. Prospective study of clinical gallbladder disease and its association with obesity, physical activity, and other factors. Dig Dis Sci. 1992;37:784-90.

19. Grodstein F, Colditz GA, Hunter DJ, Manson JE, Willett WC, Stampfer MJ. A prospective study of symptomatic gallstones in women: relation with oral contraceptives and other risk factors. Obstet Gynecol. 1994;84:207-14.

20. Sahi T, Paffenbarger RS Jr, Hsieh CC, Lee IM. Body mass index, cigarette smoking, and other characteristics as predictors of selfreported, physician-diagnosed gallbladder disease in male college alumni. Am J Epidemiol. 1998;147:644-51.

21. Liu B, Balkwill A, Roddam A, Brown A, Beral V. Separate and joint effects of alcohol and smoking on the risks of cirrhosis and gallbladder disease in middle-aged women. Am J Epidemiol. 2009;169:153-60.

22. Etminan M, Delaney JA, Bressler B, Brophy JM. Oral contraceptives and the risk of gallbladder disease: a comparative safety study. CMAJ. 2011;183:899-904.
23. Misciagna G, Leoci C, Guerra V, et al. Epidemiology of cholelithiasis in southern Italy. Part II: risk factors. Eur J Gastroenterol Hepatol. 1996;8:585-93.

24. Yamada M, Wong FL, Fujiwara S, Tatsukawa Y, Suzuki G. Smoking and alcohol habits as risk factors for benign digestive diseases in a Japanese population: the radiation effects research foundation adult health study. Digestion. 2005;71:231-7.

25. Stroup DF, Berlin JA, Morton SC, et al. Meta-analysis of observational studies in epidemiology: a proposal for reporting. Meta-analysis of observational studies in epidemiology (MOOSE) group. JAMA. 2000;283:2008-12.

26. DerSimonian R, Laird N. Meta-analysis in clinical trials. Control Clin Trials. 1986;7:177-88.

27. Greenland S, Longnecker MP. Methods for trend estimation from summarized dose-response data, with applications to meta-analysis. Am J Epidemiol. 1992;135:1301-9.

28. Aune D, Greenwood DC, Chan DS, et al. Body mass index, abdominal fatness and pancreatic cancer risk: a systematic review and non-linear dose-response meta-analysis of prospective studies. Ann Oncol. 2012;23:843-52.

29. Bagnardi V, Zambon A, Quatto P, Corrao G. Flexible meta-regression functions for modeling aggregate dose-response data, with an application to alcohol and mortality. Am J Epidemiol. 2004; 159:1077-86.

30. Higgins JP, Thompson SG. Quantifying heterogeneity in a metaanalysis. Stat Med. 2002;21:1539-58.

31. Higgins JP, Thompson SG, Deeks JJ, Altman DG. Measuring inconsistency in meta-analyses. BMJ. 2003;327:557-60.

32. Wells G, Shea B, O'Connell D. et al. The Newcastle-Ottawa Scale (NOS) for assessing the quality of nonrandomised studies in meta-analyses. http://www.ohrica/programs/clinical_epide miology/oxford.asp, Accessed 13 Aug 2014.

33. Egger M, Davey SG, Schneider M, Minder C. Bias in meta-analysis detected by a simple, graphical test. BMJ. 1997;315:629-34.

34. Begg CB, Mazumdar M. Operating characteristics of a rank correlation test for publication bias. Biometrics. 1994;50:1088-101.

35. Willi C, Bodenmann P, Ghali WA, Faris PD, Cornuz J. Active smoking and the risk of type 2 diabetes: a systematic review and meta-analysis. JAMA. 2007;298:2654-64.

36. Shebl FM, Andreotti G, Rashid A, et al. Diabetes in relation to biliary tract cancer and stones: a population-based study in Shanghai, China. Br J Cancer. 2010;103:115-9.

37. Craig WY, Palomaki GE, Haddow JE. Cigarette smoking and serum lipid and lipoprotein concentrations: an analysis of published data. BMJ. 1989;298:784-8.

38. Banim PJ, Luben RN, Bulluck H, et al. The aetiology of symptomatic gallstones quantification of the effects of obesity, alcohol and serum lipids on risk. Epidemiological and biomarker data from a UK prospective cohort study (EPIC-Norfolk). Eur J Gastroenterol Hepatol. 2011;23:733-40. 